\title{
An Atomistically Validated Continuum Model for Strain Relaxation and Misfit Dislocation Formation
}

X. W. Zhou ${ }^{1}$, D. K. Ward ${ }^{2}$, J. A. Zimmerman ${ }^{1}$, J. L. Cruz-Campa ${ }^{3}$, D. Zubia ${ }^{4}$, J. E. Martin ${ }^{5}$, and F. van $\mathrm{Swol}^{6}$

${ }^{1}$ Mechanics of Materials Department, Sandia National Laboratories, Livermore, California 94550,USA, Email: xzhou@sandia.gov

${ }^{2}$ Radiation and Nuclear Detection Materials and Analysis Department, Sandia National Laboratories, Livermore, California 94550, USA

${ }^{3}$ MEMS Technologies Department, Sandia National Laboratories, Albuquerque, New Mexico 87185, USA

${ }^{4}$ Department of Electrical Engineering, University of Texas at El Paso, El Paso, Texas 79968, $U S A$

${ }^{5}$ Nanoscale Sciences Department, Sandia National Laboratories, Albuquerque, New Mexico 87185, USA

${ }^{6}$ Computational Materials and Data Science Department, Sandia National Laboratories, Albuquerque, New Mexico 87185, USA

\begin{abstract}
In this paper, molecular dynamics (MD) calculations have been used to examine the physics behind continuum models of misfit dislocation formation and to assess the limitations and consequences of approximations made within these models. Without compromising the physics of misfit dislocations below a surface, our MD calculations consider arrays of dislocation dipoles constituting a mirror imaged "surface". This allows use of periodic boundary conditions to create
\end{abstract}


a direct correspondence between atomistic and continuum representations of dislocations, which would be difficult to achieve with free surfaces. Additionally, by using long-time averages of system properties, we have essentially reduced the errors of atomistic simulations of large systems to "zero". This enables us to deterministically compare atomistic and continuum calculations. Our work results in a robust approach that uses atomistic simulation to accurately calculate dislocation core radius and energy without the continuum boundary conditions typically assumed in the past, and the novel insight that continuum misfit dislocation models can be inaccurate when incorrect definitions of dislocation spacing and Burgers vector in latticemismatched systems are used. We show that when these insights are properly incorporated into the continuum model, the resulting energy density expression of the lattice-mismatched systems is essentially indistinguishable from the MD results.

Keywords: molecular dynamics, misfit dislocations, lattice-mismatched multilayers, semiconductor compounds

\section{INTRODUCTION}

Control of strain relaxation and misfit dislocation ${ }^{*}$ formation in thin films is a key material issue for electronic devices [1]. Misfit dislocations - line discontinuities that form at the interface between a film and a substrate in order to accommodate the mismatch between their lattice spacings - are a longstanding issue that plagues the semiconductor manufacturing industry, among others. That said, continuum models and calculations have been effective in guiding material synthesis experiments $[2,3,4,5,6,7,8,9,10,11]$ to mitigate the impact these defects have on device properties. However, as modern devices are driven towards smaller dimensions, larger

\footnotetext{
* In this paper, misfit dislocations refer generally to the edge type of dislocations that change the misfit strain regardless if these dislocations have the equilibrium density or not.
} 
lattice mismatches, and lower defect tolerances, the need for accurate continuum predictions becomes increasingly demanding. On the other hand, continuum models inherently involve approximations that may cause varying degrees of errors.

As the simplest example of an approximation, continuum theories often neglect dislocation core energies. While dislocation core energies can be easily added, accurate values of dislocation energies may not be readily available. As a second example, the Burgers vector of a misfit (edge) dislocation is essentially associated with the thickness of the half planes removed from or inserted into the system. However, inserting a half plane to the film is exactly equivalent to removing a half plane from the substrate. As a result, ambiguity arises whether the Burgers vector should be defined by the film or the substrate lattice constant. It has been suggested that an appropriate Burgers vector would enable far field stresses to become zero [12,13]. However, the far field stresses are obviously not zero in the continuous films except when a precise number of misfit dislocations are formed to cancel exactly the misfit strain. Intuitively, a Burger circuit in the strained system can be used to determine the Burgers vector $[14,15,16]$. However, an explicit proof and physics of this point has not been given in previous continuum models. In fact, it is not uncommon that the Burgers vector in past studies is taken from the lattice constant of the film $[17,18,19]$.

An additional interesting point is that the conventional continuum misfit dislocation theories often only account for the elastic properties of the film but not the substrate. While it is possible to incorporate the elastic properties of both film and substrate using more complex theories [4], the less sophisticated models can still be sufficiently accurate. A remaining question, however, is how to quantify the effects of these approximations. 
The accuracy of continuum models is often difficult to determine directly from experiments, making model improvement difficult. Fortunately, molecular dynamics (MD) simulations provide a potential, alternative approach to study strain relaxation and misfit dislocation formation $[20,21,22,23]$. However, practical devices typically have dimensions beyond $100 \mathrm{~nm}$. At such dimensions, MD models are not efficient for generating sufficient insight to impact device design and synthesis. On the other hand, MD models can be used to assess the consequences of the assumptions of the continuum models. In order to use MD to validate continuum models, MD results must match exactly continuum results given the same geometry of the problem. This is a more stringent requirement than the usual comparison between atomistic and continuum models [24] where qualitative agreement is considered sufficient.

The objective of the present paper is threefold: (a) develop an atomistic model capable of exactly matching continuum results for strain relaxation and misfit dislocation formation under the same geometry of thin film problem; (b) use this atomistic model to discover limitations of existing continuum models and propose methods for their improvement; and (c) illuminate the physics of strain relaxation and misfit dislocation formation.

\section{TOWARDS EXACT ATOMISTIC AND CONTINUUM MATCH}

Accurate atomistic calculation of energies of dislocations under the same geometry used in continuum models and a complete elimination of statistical error of large scale atomistic simulations are two challenging problems that must be overcome before an exact match between atomistic and continuum models can be achieved. For dislocation energy calculations, edge dislocations can be represented in atomistic models by removing (or inserting) extra half planes. This approach leads to different numbers of atoms in systems with and without a misfit 
dislocation. As a result, the dislocation energy is not simply the energy difference between the two systems. It is possible to introduce dislocations into simulation cells by displacing atoms rather than by changing the number of atoms. For a single dislocation, this would require the boundary of an atomistic model to be constrained at the continuum dislocation-induced displacement fields. Hence, the approach is not entirely atomistic. For a pair of positive and negative dislocations [25], no continuum displacement fields are required. However, additional constrains must be used in MD simulations to prevent the opposite dislocations from annihilation.

This problem can be resolved by using periodic boundary conditions. This is because under periodic boundary conditions, every atom in a dislocation-free system is equivalent. The energy of the dislocation-free system can then be scaled to the same number of atoms in a system containing a dislocation. Unfortunately, use of periodic boundary conditions prohibits the creation of a single dislocation. This paper seeks to address this problem by studying dislocation dipole configurations instead of isolated dislocations. Note that the particular dislocation configuration is not important as long as it can be studied in both MD and continuum models to enable the connection. By fitting continuum models to atomistic results for periodic dislocation dipole configurations, energies of individual dislocations can be accurately determined.

The numerical error associated with atomistic simulations is an extremely challenging problem. As will be shown below, conventional molecular statics (MS) energy minimization simulations do not yield unique minimum energy results when the system is large and contains dislocations [26,27]. This paper addresses the problem by calculating long time-averaged properties using MD simulations. Clearly, highly converged results can theoretically always be achieved by increasing the averaging time. In particular, previous work [27] indicates that when 
the averaging time is increased from $0.5 \mathrm{~ns}$ to $4.0 \mathrm{~ns}$, the statistic oscillation of the MD results significantly reduces but oscillation can still be visually identified from figures. However, when the averaging time is increased to $20.0 \mathrm{~ns}$, the statistic error is essentially zero. Also note that MD simulations have an additional advantage as they incorporate finite temperatures whereas the MS data pertains only to $0 \mathrm{~K}$.

As we shall show, dislocation dipole configurations and long time-averaged MD simulations are critical for an exact match between atomistic and continuum models. For simplicity and without any impact on model comparisons, our studies made two assumptions. First, as for the classical continuum model by Nix [3], we assume that the substrate is much thicker than the film. If the film/substrate interface is on the x-z plane and film thickness is in the $\mathrm{y}$ - direction, this means that the $\mathrm{x}$ - and $\mathrm{z}$ - dimensions of the substrate is fixed at its equilibrium values and only the film is strain relaxed to match the substrate. Obviously, at the absence of dislocations, the strain introduced in the film equals exactly the lattice mismatch parameter, $\varepsilon=$ $\left(a_{s}-a_{f}\right) / a_{f}$, where $a_{f}$ and $a_{s}$ are lattice constants of film and substrate respectively. Second, we assume a quasi- two-dimensional (2D) geometry. This means that we use a relatively short zdimension and do not introduce misfit dislocations (i.e., uniformly strain the film) along the zdirection. We point out that if the substrate is "infinitely" thick, fixing the dimensions to the substrate values would conceptually represent a zero overall stress of the system. The net effect of uniformly straining the film in the $\mathrm{z}$ - direction is to change its elastic constants on the $\mathrm{x}-\mathrm{y}$ plane. In fact, if this uniformly strained state is taken as the reference, then the systems under study can be viewed as satisfying a plane strain condition $\varepsilon_{\mathrm{zz}}=\gamma_{\mathrm{xz}}=\gamma_{\mathrm{yz}}=0$.

\section{CONTINUUM ENERGY OF MISFIT DISLOCATIONS}

To compare atomistic and continuum results and to reveal the associated physics, we have 
performed a rigorous derivation of a continuum model of strain relaxation and misfit dislocation formation in supplement material [28]. This continuum model assumes a "mirror imaged" film surface, rather than a true film surface. As will be demonstrated below, our mirror imaged model is similar to the classical continuum model [2,29,30,31] elegantly summarized by Nix [3] in 1989. We emphasize that since Nix, more sophisticated models accounting for true surfaces have been developed by Willis and others [4,36,37]. Our purpose is not to improve over these sophisticated models. Rather our mirror imaged surfaces allow us to study a misfit dislocation dipole array rather than a misfit dislocation array, and therefore provide an exact one-on-one correspondence between atomistic and continuum models as described above.

For connecting to existing continuum theories, we first describe Nix's model [3]. Applying Nix's equation to the 2D geometry of a lattice-mismatched film containing misfit dislocations, the energy (per unit of interface area) can be expressed as

$$
\left.E_{s}=\frac{1}{2} \frac{2 G_{f} h}{\left(1-v_{f}\right)} \cdot\left(\varepsilon-\frac{b}{S}\right)^{2}+\frac{G_{f} b^{2}}{4 \pi\left(1-v_{f}\right.}\right) \frac{1}{S} \ln \left(\frac{\beta \cdot h}{b}\right)
$$

where G, $v$, and $b$ are, respectively, shear modulus, Poisson's ratio and magnitude of the Burgers vector for a misfit dislocation (the subscript $\mathrm{f}$ standing for film), $\mathrm{S}$ is the spacing between dislocations, $\mathrm{h}$ is film thickness, and $\beta$ is a constant. Note that in Eq. (1), $\frac{2 G_{f}}{\left(1-v_{f}\right)}$ equals Young's modulus under our plane strain condition $\varepsilon_{\mathrm{xx}} \neq 0, \varepsilon_{\mathrm{yy}} \neq 0, \varepsilon_{\mathrm{zz}}=\gamma_{\mathrm{xz}}=\gamma_{\mathrm{yz}}=0$, and $\sigma_{\mathrm{yy}}=0$. Also note that Eq. (1) differs from the expression given in [3] by a factor of two because we assume a 2D problem whereas it is three-dimensional (3D) in [3].

As has been mentioned above, it is extremely important to note that in the past applications of Eq. (1), there has been some ambiguity with regard to definitions of Burgers magnitude b and dislocation spacing $\mathrm{S}$. Through a rigorous derivation, we now give precise definitions of these 
quantities. Assume that in a dislocation-free system, $\mathrm{n}_{\mathrm{f}}$ planes of film with plane spacing $\mathrm{b}_{\mathrm{f}}$ are matched with $\mathrm{n}_{\mathrm{s}}$ (assumes that $\mathrm{n}_{\mathrm{s}}=\mathrm{n}_{\mathrm{f}}$ ) planes of substrate with plane spacing $\mathrm{b}_{\mathrm{s}}$. If the substrate is rigid, then the film is subject to a mismatch strain of $\left(n_{\mathrm{f}} \mathrm{b}_{\mathrm{s}}-\mathrm{n}_{\mathrm{f}} \mathrm{b}_{\mathrm{f}}\right) /\left(\mathrm{n}_{\mathrm{f}} \mathrm{b}_{\mathrm{f}}\right)=\left(\mathrm{b}_{\mathrm{s}}-\mathrm{b}_{\mathrm{f}}\right) / \mathrm{b}_{\mathrm{f}}=\left(\mathrm{a}_{\mathrm{s}}-\mathrm{a}_{\mathrm{f}}\right) / \mathrm{a}_{\mathrm{f}}=$ $\varepsilon$. If a half plane is inserted to the film, then the film is subject to a residual strain of $\left[\mathrm{n}_{\mathrm{f}} \mathrm{b}_{\mathrm{s}^{-}}\right.$ $\left.\left(n_{\mathrm{f}}+1\right) b_{\mathrm{f}}\right] /\left[\left(\mathrm{n}_{\mathrm{f}}+1\right) \mathrm{b}_{\mathrm{f}}\right]=\varepsilon-\mathrm{b}_{\mathrm{s}} / \mathrm{S}_{\mathrm{f}}$, where $\mathrm{S}_{\mathrm{f}}=\left(\mathrm{n}_{\mathrm{f}}+1\right) \mathrm{b}_{\mathrm{f}}$ is the total length of unstrained film per dislocation (i.e., dislocation spacing measured from the film). On the other hand, if a half plane is removed from the substrate, then the film is subject to a residual strain of $\left[\left(n_{\mathrm{f}}-1\right) \mathrm{b}_{\mathrm{s}}-\mathrm{n}_{\mathrm{f}} \mathrm{b}_{\mathrm{f}}\right] /\left(\mathrm{n}_{\mathrm{f}} \mathrm{b}_{\mathrm{f}}\right)$ $=\varepsilon-b_{\mathrm{s}} / S_{f}$, where $S_{f}=n_{f} b_{f}$ is again the total length of unstrained film per dislocation. This means that regardless of whether it is viewed as inserting a half plane in the film or removing a half plane from the substrate, a dislocation always causes a consistent residual strain of $\varepsilon-b_{s} / S_{f}$, when the substrate is fixed.

By comparing the derived residual strain, $\varepsilon-\mathrm{b}_{\mathrm{s}} / \mathrm{S}_{\mathrm{f}}$, to the residual strain used on the right side of Eq. (1), $\varepsilon$ - b/S, we see that the magnitude of the Burgers vector $b$ should be the substrate value $b_{s}$ rather than the film value $b_{f}$, and dislocation spacing $S$ should be the film value $S_{f}$ rather than the substrate value $S_{s}$. In the same notion, we emphasize that the lattice mismatch parameter $\varepsilon$ should be defined as $\varepsilon=\left(a_{\mathrm{s}}-a_{\mathrm{f}}\right) / a_{\mathrm{f}}$. It is incorrect to define $\varepsilon=\left(a_{\mathrm{f}}-a_{\mathrm{s}}\right) / \mathrm{a}_{\mathrm{s}}$ by taking the substrate lattice constant as the "reference".

Physically, misfit strain comes from straining a free film to match the substrate. As a result, the dislocation spacing, which represents to sample length prior to strain, should equal $\mathrm{S}_{\mathrm{f}}$. In addition, the thickness weighted average plane spacing between a film and an infinitely thick substrate equals the substrate plane spacing. As a result, the Burgers vector should correspond to $b_{\mathrm{s}}$. Having established that the Burgers vector in misfit dislocation theory should be the substrate value $b_{s}$, we will drop the subscript $s$ in the following for convenience (i.e., $b \equiv b_{s}$ ). 
The rigorous derivation presented in our supplement material [28] produces an accurate expression for misfit dislocation energy:

$$
E_{s}=\frac{E_{c}}{S}+\frac{G_{f} b^{2}}{4 \pi\left(1-v_{f}\right)} \frac{\gamma}{S} \ln \left(\frac{h}{r_{0}}\right)+\frac{G_{f} h}{\left(1-v_{f}\right)}\left\{\gamma \frac{b^{2}}{S^{2}}(1+\varepsilon) \frac{f\left(\frac{1+\varepsilon}{S} h\right)}{4 \pi \frac{1+\varepsilon}{S} h}+\frac{\varepsilon^{2}}{1+\varepsilon}-\frac{2 \varepsilon b}{S}\right\}
$$

Here $E_{c}$ is dislocation core energy, $\mathrm{G}_{\mathrm{f}}$ and $v_{\mathrm{f}}$ are shear modulus and Poisson's ratio of the film, $\gamma$ is a parameter correcting the error due to different elastic constants of film and substrate (i.e., $\gamma=$ 1 when the film and the substrate have the same elastic constants), and $f(x)$ is expressed as

$$
f(x)=2 \pi \cdot x \cdot \operatorname{coth}(2 \pi \cdot x)+\ln [\sinh (2 \pi \cdot x)]-\ln (2 \pi \cdot x)-1
$$

where coth, sinh (and cosh to be referred below) are hyperbolic functions. Eq. (2) is similar to the result from Willis et al [4,36,37], although subtle difference exists as our expression is precise for a mirror imaged surface whereas theirs is for a true surface.

Note that the function $f(x)$ can be approximated by $4 \pi x$ [28]. If $f(x)$ is replaced by $4 \pi x$, our energy expression can be written as

$$
E_{s}=\frac{E_{c}}{S}+\frac{G_{f} b^{2}}{4 \pi\left(1-v_{f}\right)} \frac{\gamma}{S} \ln \left(\frac{h}{r_{0}}\right)+\frac{G_{f} h}{\left(1-v_{f}\right)}\left[\gamma \frac{b^{2}}{S^{2}}(1+\varepsilon)+\frac{\varepsilon^{2}}{1+\varepsilon}-\frac{2 \varepsilon b}{S}\right]
$$

It can be seen from Eq. (4) that if we ignore the dislocation core energy $E_{c}$, set $\gamma=1$, and

recognize that $\mathrm{S}_{\mathrm{f}}=\mathrm{S} /(1+\varepsilon)$, Eq. (4) becomes $E_{s}=\frac{1}{2} \frac{2 G_{f} h}{\left(1-v_{f}\right)} \cdot \frac{S_{f}}{S}\left(\varepsilon-\frac{b}{S_{f}}\right)^{2}+\frac{G_{f} b^{2}}{4 \pi\left(1-v_{f}\right)} \frac{1}{S} \ln \left(\frac{h}{r_{0}}\right)$. This is essentially the same as Eq. (1) because the parameter $b_{f} / \beta$ can be simply considered as equivalent to $\mathrm{r}_{0}$, and $\mathrm{S}$ in Eq. (1) can be considered (approximately) as $\mathrm{S}_{\mathrm{f}}$. Discussion on the validity of the approximation of $4 \pi \mathrm{x}$ for $\mathrm{f}(\mathrm{x})$ is presented in supplemental material [28]. 
Our derivation [28] reveals some physical insights. In particular, we find from Eq. (S4) of supplement material [28] that the dislocation line energy is $\Gamma=E_{c}+\frac{G_{f} b^{2}}{4 \pi\left(1-v_{f}\right)} \gamma\left[\ln \left(\frac{h}{r_{0}}\right)+f\left(\frac{h}{S_{f}}\right)\right] \approx$ $E_{c}+\frac{G_{f} b^{2}}{4 \pi\left(1-v_{f}\right)} \gamma \ln \left(\frac{h}{r_{0}}\right)+\frac{G_{f} b^{2}}{\left(1-v_{f}\right)} \gamma \frac{h}{S_{f}}$. Clearly $\frac{G_{f} b^{2}}{4 \pi\left(1-v_{f}\right)} \gamma \ln \left(\frac{h}{r_{0}}\right)$ is dislocation self-energy because it is equivalent to $\Gamma$ in the isolated dislocation condition $\mathrm{S}_{\mathrm{f}} \rightarrow \infty$. The additional term $\frac{G_{f} b^{2}}{\left(1-v_{f}\right)} \gamma \frac{h}{S_{f}}$ in the $\Gamma$ expression then represents the interactive energy [16] of different dislocations in the array.

It can also be seen that for a homogeneous material system (i.e., the system contains dislocations but the mismatch strain $\varepsilon=0$, and $\gamma=1$ ), Eq. (4) correctly reduces to dislocation energy per unit of surface area, i.e., $\Gamma$ divided by $S$. Furthermore, this dislocation energy is a summation of dislocation self-energy $\frac{E_{c}}{S}+\frac{G_{f} b^{2}}{4 \pi\left(1-v_{f}\right)} \frac{1}{S} \ln \left(\frac{h}{r_{0}}\right)$, and a residual strain energy of $\frac{1}{2} \cdot E_{f} \cdot \varepsilon_{d}^{2} \cdot \frac{V}{A}$, where $E_{f}=\frac{2 G_{f}}{1-v_{f}}$ is the Young's modulus of the film, $\varepsilon_{d}=\frac{b}{S_{f}}$ is the strain caused by the dislocations, $V=h \cdot S_{f}$ is the film volume per dislocation, and $\mathrm{A}=\mathrm{S}$ is the interfacial area per dislocation. This residual strain energy accounts for the interactive energy of dislocations, and is consistent with the notion that the overlap of the elastic field of an array of edge dislocations essentially leads to a uniform strain $\varepsilon_{d}$ in the far field.

In homogeneous systems, the residual strain energy is essentially the interactive energy of dislocations and the total energy reduces to dislocation energy. These consistencies are not apparent from Nix's original description [3]. This does not mean that Nix's model is fundamentally different from ours. It just means that in Nix's model, dislocation energy refers 
only to the dislocation self-energy whereas in our model, dislocation energy includes both dislocation self-energy and interactive energy between dislocations.

\section{CONTINUUM ENERGY OF DISLOCATION DIPOLES UNDER FULL PERIODIC BOUNDARY CONDITIONS}

Eq. (2) derived above can be used to calculate energy of a film/substrate structure, but not energy of periodic dislocation dipoles ${ }^{\dagger}$. As described above, atomistic simulations can be used to calculate accurate dislocation energies using dipole configurations under periodic boundary conditions. In order to independently validate dislocation energy, a continuum energy expression for periodic dislocation dipoles has been derived in supplement material [28]. We note that similar continuum models have been explored previously [38], albeit no explicit energy expression is given due to a conditional convergence issue. For our geometry, no conditional convergence issue occurs. According to the derivation [28], the dislocation energy under the periodic boundary conditions is

$\Gamma=E_{0}+2 \sum_{i=1}^{\infty} E_{i}$

where

$E_{0}=E_{c}+\frac{G b^{2}}{4 \pi(1-v)}\left\{\ln \left(\frac{d}{2 r_{0}}\right)+\ln \left(\frac{L_{y}-d}{L_{y}-2 r_{0}}\right)-\ln \left[G a\left(\frac{L_{y}+d}{L_{y}}\right)\right]-\ln \left[G a\left(2-\frac{d}{L_{y}}\right)\right]\right\}$

and

\footnotetext{
${ }^{\dagger}$ See Fig. S4(a) of supplement material [28] for schematic illustration of the periodic dislocation dipole configuration.
} 


$$
E_{i}=\frac{G b^{2}}{4 \pi(1-v)}\left\{\begin{array}{l}
\frac{2 \pi \cdot i \cdot L_{x} \cdot \operatorname{Coth}\left(\frac{\pi \cdot i \cdot L_{x}}{L_{y}}\right) \cdot \operatorname{Sin}^{2}\left(\frac{\pi \cdot d}{L_{y}}\right)}{L_{y} \cdot \operatorname{Cosh}\left(\frac{2 \pi \cdot i \cdot L_{x}}{L_{y}}\right)-L_{y} \cdot \operatorname{Cos}\left(\frac{2 \pi \cdot d}{L_{y}}\right)}+ \\
\frac{1}{2} \ln \left[\operatorname{Cos}^{2}\left(\frac{\pi \cdot d}{L_{y}}\right)+\operatorname{Coth}^{2}\left(\frac{\pi \cdot i \cdot L_{x}}{L_{y}}\right) \cdot \operatorname{Sin}^{2}\left(\frac{\pi \cdot d}{L_{y}}\right)\right]
\end{array}\right\}
$$

Eqs. (5) - (7) fully define dislocation energy as a function of periodic length in the $\mathrm{x}$ - direction $\mathrm{L}_{\mathrm{x}}$, periodic length in the $\mathrm{y}$ - direction $\mathrm{L}_{\mathrm{y}}$, and dislocation dipole distance d. Eqs. (5) - (7), however, involve three unknown parameters, namely dislocation core energy $\mathrm{E}_{\mathrm{c}}$, core radius $\mathrm{r}_{0}$, and elastic constant ratio $\mathrm{G} /(1-\mathrm{v})$.

\section{MD GEOMETRIES OF SIMULATIONS, AND CALCULATIONS OF LATTICE AND ELASTIC CONSTANTS OF OUR MODEL SYSTEM}

Eq. (2) is a rigorous continuum energy expression for the energy of a lattice-mismatched system as a function of number of misfit dislocations. It is composed of dislocation energy and residual misfit strain energy. Eqs. (5)-(7) provide a rigorous continuum energy expression for dislocation arrays. Residual strain energy volume density $\mathrm{E}_{\varepsilon}$ can be accurately described by the linear elastic law $\mathrm{E}_{\varepsilon}=1 / 2 \mathrm{E} \cdot \varepsilon_{\mathrm{r}}{ }^{2}$ where $\mathrm{E}$ is an elastic constant (e.g., Young's modulus) and $\varepsilon_{\mathrm{r}}$ is the residual strain. To clearly reveal the physics of Eq. (2), we will use atomistic simulations to not only match Eq. (2), but also independently match Eq. (5) and the linear elastic law.

\section{A. Simulation Geometries}

We first describe the MD geometries compatible with those behind Eqs. (2) and (5). As a generic example, here we use the Cd-Te-S Stillinger-Weber potential [39] to model the growth of CdTe on a thick (i.e., non-compliant) CdS substrate. This stated, we note that the particular 
materials are not important because our objective is to compare MD and continuum models.

Thus, any lattice-mismatched system will be sufficient.

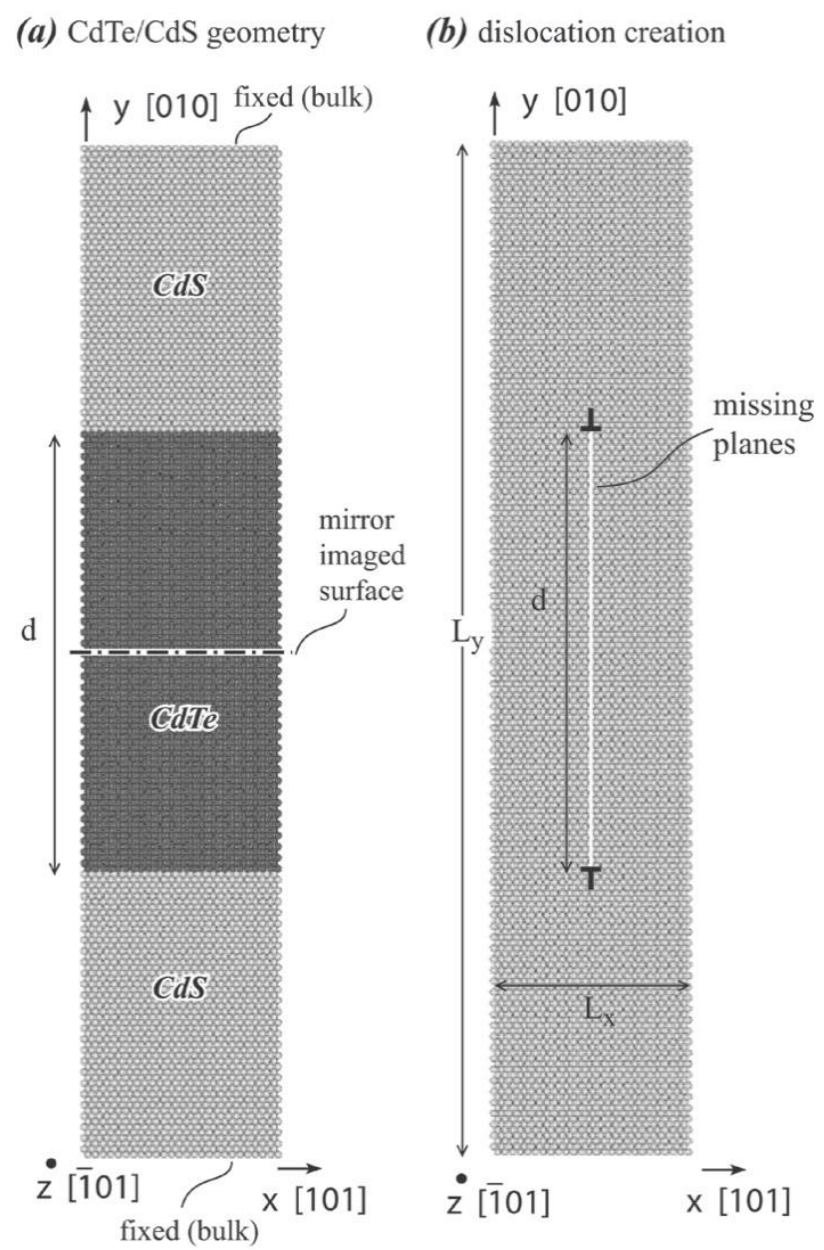

Fig. 1. (a) MD Geometry of the CdTe on CdS structures where CdS is fixed in the $\mathrm{x}$ - and zdirections to the equilibrium bulk sizes to mimic a very large CdS thickness. The dotteddashed line mimics the mirror imaged "surface"; and (b) creation of misfit dislocation dipoles (dislocation and its image) by removing planes within a thickness of Burgers magnitude b. Note that geometry shown in (b) is used for both inhomogeneous $(\mathrm{CdTe} / \mathrm{CdS})$ and homogeneous (pure CdTe and pure CdS) systems. The inhomogeneous system can be used to validate Eq. (2). The homogeneous systems are for dislocation energy calculations aiming to validate Eqs. (5)-(7), where only the z- direction is fixed to the equilibrium bulk size of $\mathrm{CdS}$ and both $\mathrm{x}$ - and $\mathrm{y}$ - directions are relaxed.

The geometry of the CdTe/CdS periodic system is shown in Fig. 1(a), where $\mathrm{x}_{-}^{-}, \mathrm{y}^{-}$, and zare aligned with [101], [010], and [ $\overline{101}$ ] respectively, and the (010) CdTe/CdS interface is 
parallel to the $\mathrm{x}-\mathrm{z}$ plane. For the $2 \mathrm{D}$ case, we assume that the $\mathrm{z}$ - dimension of the system is fixed at the equilibrium lattice size of $\mathrm{CdS}$, and hence, strain relaxation only occurs in the $\mathrm{x}$ - direction. To create misfit dislocation dipoles, some atomic planes (with a total thickness of a Burgers magnitude b), as indicated by the white line in Fig. 1(b), are removed. It can be seen that under the periodic boundary conditions, the geometry in Fig. 1(b) is exactly the same as that in the continuum model [28].

\section{B. MD Calculations of Lattice Constants}

Molecular statics calculations of CdTe and CdS bulk crystals indicate that the CdS and CdTe lattice constants at $0 \mathrm{~K}$ are $5.835 \AA$ and $6.478 \AA$, respectively. Performing MD simulations at $300 \mathrm{~K}$ for $4 \mathrm{~ns}$ to equilibrate the systems, and another $8 \mathrm{~ns}$ to calculate average quantities, we find that the CdS and CdTe equilibrium lattice constants at $300 \mathrm{~K}$ are $5.847 \pm 0.000 \AA$ and 6.491 $\pm 0.000 \AA$, respectively. Here the average lattice constants obtained at various times are used to estimate the statistic deviation of the result. The MD results averaged over the $8 \mathrm{~ns}$ span give highly converged results with essentially zero standard error up to at least the third decimal point.

The lattice constants determined above are for the 3D systems. For our 2D case where the orientation is shown in Fig. 1 and the $\mathrm{z}$ - dimension is fixed at the $3 \mathrm{D}$ CdS size (i.e., $\mathrm{d}_{(\overline{1} 01)}=$ $5.847 / \sqrt{2}$ ), the systems may deviate from the cubic symmetry especially for CdTe where the $\mathrm{z}$ dimension is now highly compressed. As a result, the $\mathrm{x}$ - and $\mathrm{y}$ - dimensions may correspond to different lattice constants. A second set of MD simulations are performed to calculate the equilibrium CdTe and CdS lattice constants in the $\mathrm{x}$ - direction under the 2D condition, again using an equilibration time of $4 \mathrm{~ns}$ and an averaging time of $8 \mathrm{~ns}$. Note that the lattice constant in the $y$-direction is not explored because it is irrelevant to the lattice mismatch. We find that when 
the $\mathrm{z}$ - dimension is constrained at the $\mathrm{d}_{(\overline{1} 01)}=5.847 / \sqrt{2}$, the $300 \mathrm{~K} \mathrm{CdS}$ and CdTe lattice

constants in the $\mathrm{x}$ - direction (from $\mathrm{a}=\sqrt{2} \mathrm{~d}_{(101)}$ ) change to $5.769 \pm 0.000 \AA$ and $6.557 \pm 0.000 \AA$, respectively. This means that under the $2 \mathrm{D}$ condition, our $\mathrm{CdTe} / \mathrm{CdS}$ system corresponds to a lattice mismatch of $\varepsilon=(5.769-6.557) / 6.557 \approx-12.0177 \%$.

\section{MD Calculations of Young's Modulus}

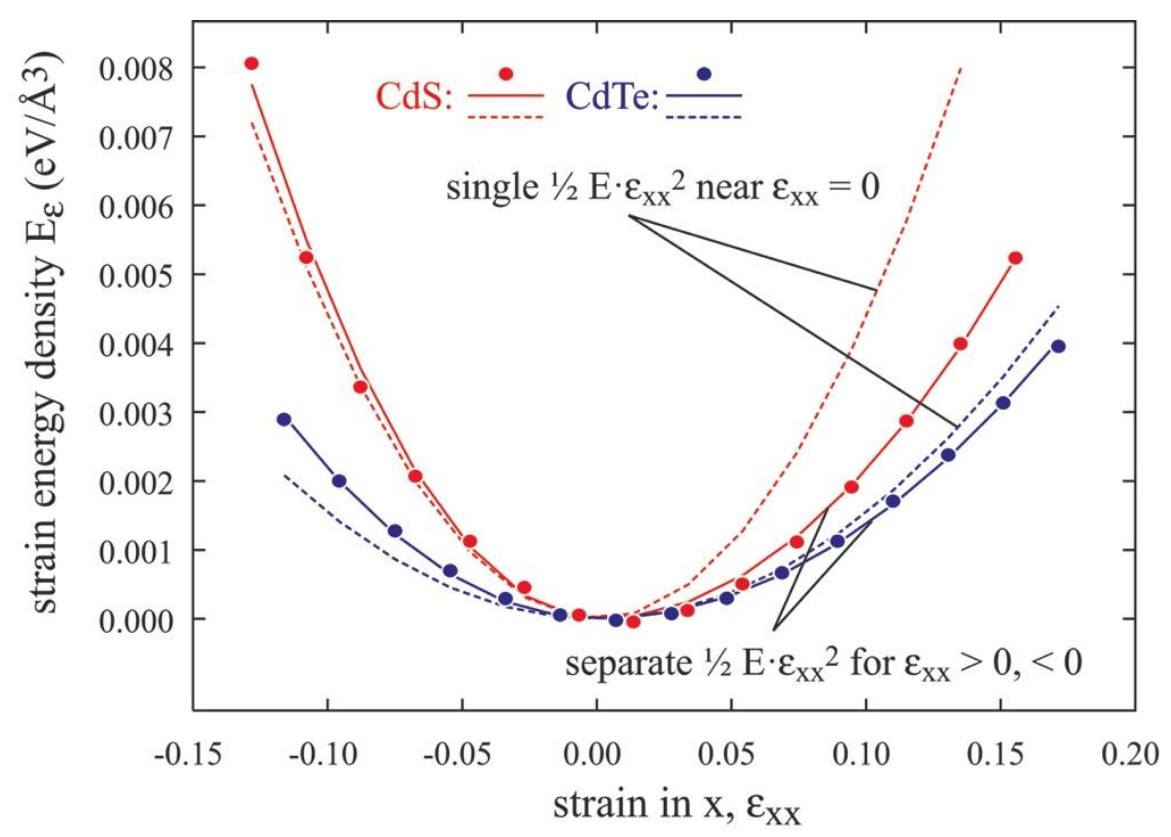

Fig. 2. Strain energy density of CdS (red) and CdTe (blue). Dash lines are fitted to linear elasticity theory over a small strain range near equilibrium. Solid lines are also fitted to linear elasticity theory, except that positive and negative strain ranges are fitted independently.

We now validate that MD results match the linear elastic law. With the orientation given in Fig. 1 and the z-dimension fixed at the 3D CdS size, the 2D CdS and CdTe bulk crystals are uniaxially deformed in the $\mathrm{x}$ - direction to various strains $-0.13<\varepsilon_{\mathrm{xx}}<0.18$, where positive strain means tension and negative strain indicates compression. Using an equilibration time of 4 ns and an averaging time of $8 \mathrm{~ns}$, similar 2D MD simulations as described above are performed to calculate the $300 \mathrm{~K}$ strain energy density $\mathrm{E}_{\varepsilon}$ for both CdS and CdTe as a function of $\varepsilon_{\mathrm{xx}}$ (here the 
$\mathrm{x}$ - dimension is also fixed leaving only the strain in the $\mathrm{y}$ - direction relaxed). The results are shown in Fig. 2, where red and blue colors distinguish CdS and CdTe. The dashed lines are fitted to linear elastic theory $\mathrm{E}_{\varepsilon}=1 / 2 \mathrm{E} \cdot \varepsilon_{x x}^{2}$ for a small strain range near equilibrium $\left(\varepsilon_{\mathrm{xx}}=0\right)$. The solid lines are also fitted to $\mathrm{E}_{\varepsilon}=1 / 2 \mathrm{E} \cdot \varepsilon_{x x}^{2}$ except that the positive and negative strain ranges are fitted independently.

Fig. 2 shows that the strain energy obtained from MD simulations approximately satisfies the linear elasticity relation $\mathrm{E}_{\varepsilon}=1 / 2 \mathrm{E} \cdot \varepsilon_{x x}^{2}$ only within a very small strain range (dash lines). We also observe that deviations from this approximation are asymmetric with regard to positive versus negative strain. For CdS, the approximation appears to be valid for large compressive strains, but only very small tensile ones. In the latter regime, the material is far less stiff than a linear model would predict. Similarly, for CdTe this asymmetry is much less pronounced, but it is nonetheless clear that the linear model over-predicts the modulus in the tensile strain regime and under-predicts it in the compressive strain regime.

It is clear that the quadratic approximation for these material's hyper-elastic strain energy density functions is only valid for a small strain range $(\sim \pm 2 \%)$, a more limited range than of interest here. As noted earlier, the true mathematic representation of these functions are no doubt complex, and would make the force/energy analysis performed here tremendously more difficult. Fortunately, only Young's modulus in the film is important in our problem. Because our CdTe film is only subjected to compression but not tension, we can use the CdTe modulus fitted to the compressive regime of the MD data to accurately describe the elastic behavior of the material over a large strain range, as shown in Fig. 2. For this consideration, we give values of Young's modulus fitted to different regimes: for the 2D case studied here, we find that the CdS Young's modulus is $\mathrm{E}=0.4323 \mathrm{eV} / \AA^{3}$ for positive strains and $\mathrm{E}=0.9399 \mathrm{eV} / \AA^{3}$ for negative strains, and 
the CdTe Young's modulus is $\mathrm{E}=0.2738 \mathrm{eV} / \AA^{3}$ for positive strains and $\mathrm{E}=0.4358 \mathrm{eV} / \AA^{3}$ for negative strains. If a single elastic constant is assumed to fit the data, then we found $\mathrm{E}=0.8724$ $\mathrm{eV} / \AA^{3}$ for $\mathrm{CdS}$ and $\mathrm{E}=0.3071 \mathrm{eV} / \AA^{3}$ for CdTe.

\section{MD CALCULATIONS OF CdTe AND CdS DISLOCATION ENERGIES UNDER PERIODIC BOUNDARY CONDITIONS}

\section{A. Effect of System Size}

We now validate Eqs. (5)-(7). Based on the orientation shown in Fig. 1, it is convenient to use the number of atomic planes $\mathrm{n}_{\mathrm{x}}, \mathrm{n}_{\mathrm{y}}$, and $\mathrm{n}_{\mathrm{z}}$ to represent the dimensions in the three coordinate directions $\mathrm{n}_{\mathrm{x}}=L_{x} / d_{\{101\}}, \mathrm{n}_{\mathrm{y}}=L_{y} / d_{\{100\}}$, and $\mathrm{n}_{\mathrm{z}}=L_{z} / d_{\{101\}}$, where $d_{\{101\}}$ and $d_{\{100\}}$ are the spacing between the corresponding atomic planes $\{\mathrm{hkl}\}$. For the $2 \mathrm{D}$ case, only a small dimension in the

z- direction is needed, and hence we use a constant $n_{z}=6\left(L_{z} \sim 25 \AA\right)$ throughout our study. With that given, we first explore the effect of system size on dislocation energy using $\mathrm{CdS}$ as an example. Dislocation dipoles are created by removing the corresponding planes as shown in Fig. 1(b). Two dislocation dipole distances $\mathrm{d}=40 d_{\{100\}}(\sim 230 \AA)$ and $\mathrm{d}=60 d_{\{100\}}(\sim 350 \AA)$ are studied. For each dislocation dipole distance, 10 system dimensions corresponding to $\mathrm{n}_{\mathrm{x}} \times \mathrm{n}_{\mathrm{y}}=$ $24 \times 86,26 \times 92,28 \times 98,30 \times 104,32 \times 110,34 \times 116,36 \times 122,38 \times 128,40 \times 134$, and $42 \times 140$ are used. Here the smallest system $n_{x} \times n_{y}=24 \times 86$ corresponds to $L_{x} \times L_{y} \sim 100 \times 500 \AA^{2}$, whereas the largest system $n_{x} \times n_{y}=42 \times 140$ corresponds to $L_{x} \times L_{y} \sim 170 \times 820 \AA^{2}$. With the chosen system dimensions, $\mathrm{L}_{\mathrm{y}}$ and $\mathrm{L}_{\mathrm{x}}$ roughly satisfy the relation $\mathrm{L}_{\mathrm{y}}=81.7(\AA)+4.24 \mathrm{~L}_{\mathrm{x}}$.

MD simulations are performed at $300 \mathrm{~K}$ for $4 \mathrm{~ns}$ to equilibrate the systems, and another 16 ns to calculate average energies of both perfect crystals and crystals containing the dislocation 
dipoles. If the energies for the perfect and the dislocated crystals are denoted as $\mathrm{E}_{\mathrm{p}}$ and $\mathrm{E}_{\mathrm{d}}$, respectively, then the dislocation line energy is calculated as

$$
\Gamma=\frac{E_{d}-\frac{N_{d}}{N_{p}} \cdot E_{p}}{2 \cdot L_{z}}
$$

where $\mathrm{N}_{\mathrm{d}}$ and $\mathrm{N}_{\mathrm{p}}$ are respectively total number of atoms in the dislocated and perfect systems (the ratio $\mathrm{N}_{\mathrm{d}} / \mathrm{N}_{\mathrm{p}}$ scales the perfect energy towards the same number of atoms in the dislocated system), and $2 \cdot \mathrm{L}_{\mathrm{z}}$ is the total length of the dislocations. The results of the MD simulations are shown in Fig. 3 as the circular data points, where the lines are fitted to Eqs. (5)-(7). Note that Eqs. (5)-(7) involve some parameters such as dislocation core radius $r_{0}$, core energies $E_{c}$, and the Young's modulus $E=\frac{2 G}{1-v}$. All three parameters, $\mathrm{r}_{0}, \mathrm{E}_{\mathrm{c}}$, and $\mathrm{E}$, are not fitted to the MD data in Fig. 3, but are rather fitted to independent MD data to be described below. Hence, the excellent agreement between the MD data and the line is a strong validation of the consistence between the continuum theory and the MD simulations.

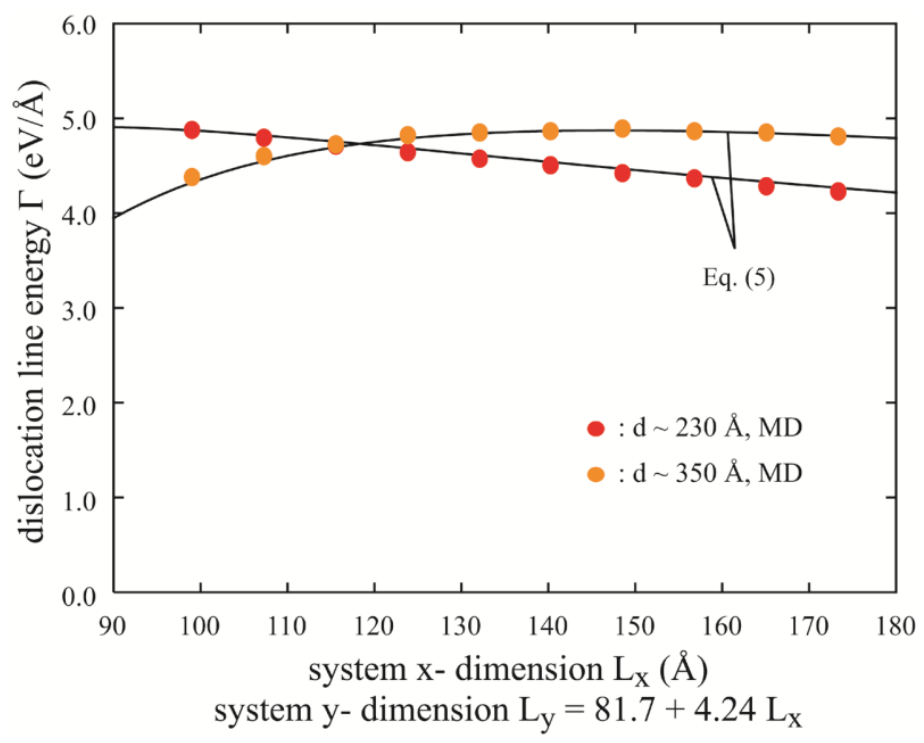

Fig. 3. CdS dislocation line energy as a function of system dimension. 
Fig. 3 indicates that average MD calculations with an average time of $16 \mathrm{~ns}$ produce highly accurate results, in agreement with previous work [27]. For comparison, molecular statics simulations are also performed to calculate the dislocation energies for the case with dipole distance $\mathrm{d}=40 d_{\{100\}}(\sim 230 \AA)$. We find that only when the system is small, MS can achieve results reasonably close to MD. Otherwise MS creates significant statistic (positive and negative) errors that increase with system sizes. In fact, the scatter is so large that the MS data does not form a trend and therefore cannot be used for validating continuum models. These results agree with past works that have examined MS simulations of large or defective systems [26,27], and are likely to be caused by the trapping of energy minimization routine due to truncation error of numerical data that can sum up to large values in large systems. In addition, the presence of dislocations allows the system to relax to many different local energy minima. The difficulties are overcome in average MD calculations where numerical truncation errors are statistically averaged out during dynamical processes and different energy minima can all be sampled according to the Boltzmann's energy distribution. We emphasize that we have tried various approaches to help more thoroughly minimize the energy in our MS simulations, including many alternating MD and MS steps at various MD temperatures. Hence, it is clear that MS simulations cannot lead to unique results for large systems (e.g., the largest system explored here involves 140,160 atoms) containing defects, and without the high quality MD data, the present work would not have been possible.

For the $\mathrm{d} \sim 230 \AA$ case, both the data points and line in Fig. 3 indicate a monotonically decreasing dislocation line energy with system dimension. Considering that the MD data is highly converged and the line represents a continuum model, this monotonic trend is real. Additional simulations with an order of magnitude increase in the dimensions confirmed that 
dislocation line energies are significantly lower than the ones shown in Fig. 3. Intuitively, dislocation energy reaches a maximum when opposite dislocations are most widely separated so that their elastic fields have the least amount of overlap (cancel). Under the periodic boundary condition, the widest dislocation separation occurs when dislocation dipole distance $\mathrm{d}$ reaches $\mathrm{L}_{\mathrm{y}} / 2$. When the spacing $\mathrm{d}$ between a dislocation and one of its neighbor reaches $\mathrm{d}>\mathrm{L}_{\mathrm{y}} / 2$, the spacing between this dislocation and its other neighbor becomes $d^{\prime}=L_{y}-d<L_{y} / 2$. Based on this recognition, when $d=230 \AA$, the maximum dislocation energy occurs at $L_{y}=460 \AA$, which gives $L_{x}=89 \AA$ according to our constraint of the $L_{y}$ vs. $L_{x}$ relation. This $L_{x}$ is lower than the dimension range explored in Fig. 3, and hence the dislocation energy for the $d=230 \AA$ case is seen to monotonically decrease in Fig. 3. When $d=350 \AA$, the maximum dislocation energy occurs at $\mathrm{L}_{\mathrm{y}}=700 \AA$, which gives $\mathrm{L}_{\mathrm{x}}=146 \AA$. Indeed, the maximum dislocation energy for the $\mathrm{d}$ $=350 \AA$ case seems to occur between $\mathrm{L}_{\mathrm{x}}=140 \AA$ and $\mathrm{L}_{\mathrm{x}}=150 \AA$.

\section{B. Effect of Dislocation Dipole Distance}

Now we further validate Eqs. (5)-(7) by exploring dislocation energy as a function of dipole distance at fixed system dimensions of $n_{x} \times n_{y}=32 \times 110$. The same MD methods as described above are used to calculate the dislocation energies of the CdS and CdTe 2D crystals at 10 different dislocation dipole spacing of $\mathrm{d}=10 d_{\{100\}}, 20 d_{\{100\}}, 30 d_{\{100\}}, 40 d_{\{100\}}, 50 d_{\{100\}}, 60$ $d_{\{100\}}, 70 d_{\{100\}}, 80 d_{\{100\}}, 90 d_{\{100\}}$ and $100 d_{\{100\}}$. The results are shown in Fig. 4 using the circular data points. The MD data is also used to fit the unknown parameters in the continuum expression, Eqs. (5)-(7), namely, dislocation core radius $r_{0}$, core energies $E_{c}$, and Young's modulus $\mathrm{E}=\frac{2 G}{1-v}$. The fitted parameters are shown in Table I. Note that Young's moduli of $\mathrm{CdS}$ and CdTe have also been determined from independent MD simulation of uniaxial 
deformation as described in Fig. 2. The Young's moduli from the uniaxial deformation simulations are included in Table I. It can be seen that the Young's modulus derived from the dislocation energy simulations is between the compressive and tensile Young's moduli determined from the deformation simulations, confirming the consistency of the calculations.

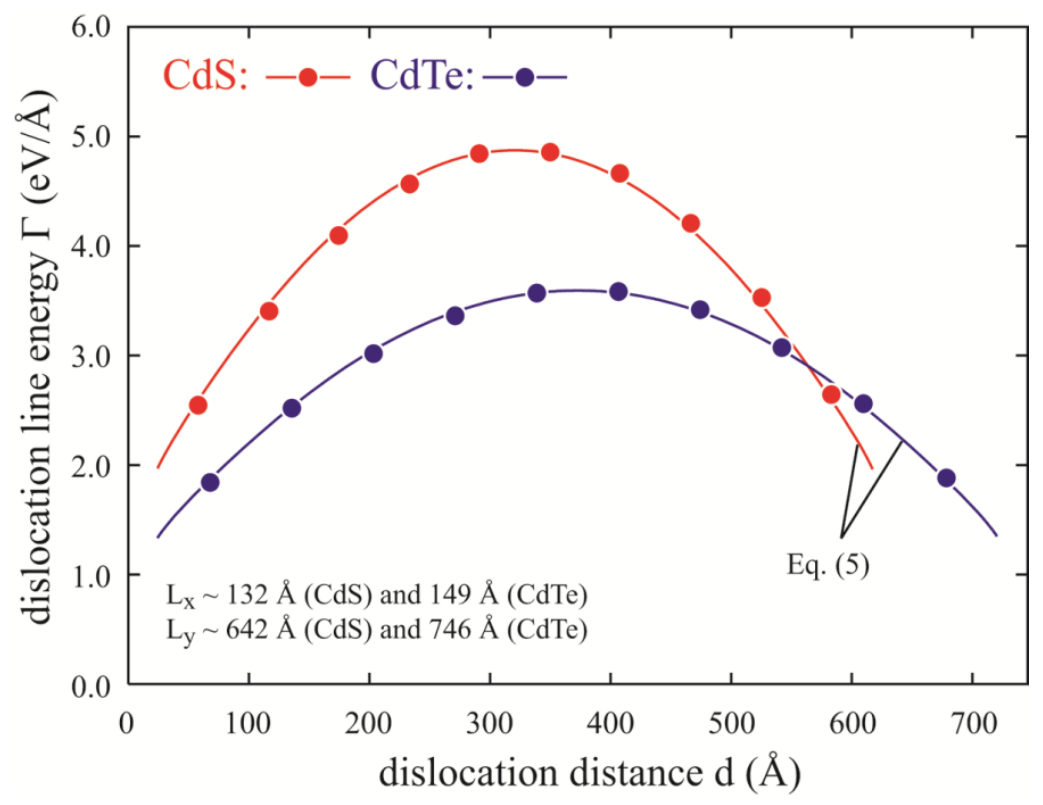

Fig. 4. CdS and CdTe dislocation energies as a function of dislocation dipole distance for given system dimensions $\mathrm{L}_{\mathrm{x}}$ and $\mathrm{L}_{\mathrm{y}}$.

Table I. Dislocation core radius $\mathrm{r}_{0}(\AA)$, core energy $\mathrm{E}_{\mathrm{c}}(\mathrm{eV} / \AA)$, and apparent Young's modulus $\mathrm{E}$ $\left(\mathrm{eV} / \AA^{3}\right)$ as derived from MD dislocation energy and MD uniaxial deformation simulations.

\begin{tabular}{|c|r|c|c|c|c|c|}
\hline \multirow{2}{*}{ material } & \multicolumn{2}{|c|}{ dislocation energy simulations } & \multicolumn{3}{c|}{ Uniaxial deformation simulations } \\
\cline { 2 - 7 } & $\mathrm{r}_{0}$ & $\mathrm{E}_{\mathrm{c}}$ & $\begin{array}{c}\mathrm{E}= \\
2 \mathrm{G} /(1-\mathrm{v})\end{array}$ & \multicolumn{3}{|c|}{$\mathrm{E}=2 \mathrm{G} /(1-\mathrm{v})$} \\
\cline { 5 - 7 } & & & compression & tension & overall \\
\hline $\mathrm{CdS}$ & 9.3141 & 1.7748 & 0.6894 & 0.9399 & 0.4323 & 0.8724 \\
\hline $\mathrm{CdTe}$ & 12.4596 & 1.2984 & 0.3921 & 0.4358 & 0.2738 & 0.3071 \\
\hline
\end{tabular}

We point out that strictly speaking, there are no unique solutions for dislocation core radius and energy. This is because by concept, any radius can be taken as the core radius as long as the materials beyond this radius follow the linear elastic theory. Obviously a large radius always 
satisfies this condition. For practical reasons, a small core radius is preferred to minimize the effect of ignoring the core in the classical elastic theory. Ideally, one would constrain the core radius to progressively smaller values and fit the other parameters. The smallest radius that yields a satisfactory fit can be taken as the ideal radius. Our core radii from un-constrained fits are acceptable as they are not too big, and both our core radii and core energies are consistent with the values typically assumed in literature [40]. Most importantly, we do not ignore the core contribution in our model so that even a big radius does not introduce errors.

The fitted continuum curves are included in Fig. 4 as represented by lines. An excellent agreement between MD and continuum calculations is obtained, supporting that the rigorously derived continuum model incorporates the physics needed to describe the dislocation energy under the periodic boundary conditions. Note that the lines in Fig. 3 are also calculated with the same set of parameters fitted for Fig. 4. While the agreement achieved in Fig. 4 may be directly attributed to the parameter optimization, the agreement achieved in Fig. 3 is not a direct result of the fitting.

Fig. 4 indicates that dislocation energy as a function of dislocation dipole distance $\mathrm{d}$ reaches a maximum and is symmetric at $\mathrm{d}=\mathrm{L}_{\mathrm{y}} / 2$. This can be easily understood because under the periodic boundary conditions, our dislocation dipole geometry can be described by Fig. 1(b). Increasing the dipole distance is equivalent to moving the positive (upper) dislocation array in the $y$ - direction while keeping the negative (lower) dislocations fixed. Each positive dislocation can be viewed as forming two dipoles, one with a negative dislocation below at a distance $\mathrm{d}$, and the other one with a negative (image) dislocation above with a distance $\mathrm{L}_{\mathrm{y}}-\mathrm{d}$. Hence, the overall energy is symmetric with the symmetric point occurring at $d=L_{y} / 2$. 


\section{EXAMINATION OF CONTNUUM MISFIT DISLOCATION THEORY USING MD SIMULATIONS}

Figures 2-4 show that MD simulations can reproduce exactly the continuum strain energies and dislocation energies that are all the physics involved in the continuum model, Eq. (2). It is therefore absolutely clear that any differences between Eq. (2) and the corresponding MD simulations would indicate some conceptual inconsistencies of the continuum model rather than numerical errors. MD simulations are therefore carried out to calculate energies of latticemismatched systems. Using the geometry shown in Fig. 1(a), CdS crystals with 32 (101) planes in the $\mathrm{x}$ - direction, $110(010)$ planes in the $\mathrm{y}$ - direction, and $6(\overline{1} 01)$ planes in the z-direction, are created. As described above, the $\mathrm{x}$ - and $\mathrm{z}$ - dimensions are fixed at the CdS sizes (i.e., $d_{(101)}=$ $5.769 / \sqrt{2}$, and $\left.d_{(\overline{1} 01)}=5.847 / \sqrt{2}\right)$. The middle region of the CdS crystal of various thicknesses, $\mathrm{d}=2 \mathrm{~h}=10 d_{(010)}, 20 d_{(010)}, 30 d_{(010)}, 40 d_{(010)}, 50 d_{(010)}$, and $60 d_{(010)}$, are changed to CdTe Misfit dislocation dipoles are created by removing extra planes in the CdTe region, as shown in Fig. 1(b). Zero, one, two, and four dislocation dipoles are all simulated. If no dislocation dipoles are created, then the dislocation spacing $\mathrm{S}$ is infinite. If the system contains $\mathrm{m}$ dislocation dipoles, the dislocation spacing is $\mathrm{L}_{\mathrm{x}} / \mathrm{m}$. Hence, creation of 1,2 , and 4 dislocation dipoles give $\mathrm{S}$ $=32 d_{(010)}(130 \AA), 16 d_{(010)}(65 \AA)$, and $8 d_{(010)}(33 \AA)$ respectively.

MD simulations are performed at $300 \mathrm{~K}$ for $8 \mathrm{~ns}$ to equilibrate the systems, and another 16 ns to calculate average energies. The energies obtained from MD simulations include cohesive energies of atoms, and extra energies from the $\mathrm{CdTe} / \mathrm{CdS}$ interfaces. Both these energy terms are not included in Eq. (2), and hence need to be removed. If the number of Te and $\mathrm{S}$ atoms in the system are $\mathrm{N}_{\mathrm{Te}}$ and $\mathrm{N}_{\mathrm{S}}$ respectively, then the number of Cd atoms $\mathrm{N}_{\mathrm{Cd}}=\mathrm{N}_{\mathrm{Te}}+\mathrm{N}_{\mathrm{S}}$. If the total 
energy of the system is $\mathrm{E}_{\mathrm{tot}}$, the cohesive energies (per atom) of CdTe and CdS bulk crystals are $\mathrm{E}_{\mathrm{CdTe}}$ and $\mathrm{E}_{\mathrm{CdS}}$ respectively, and the interfacial energy is $\rho$, then the MD version of the energy defined by Eq. (2) becomes

$$
E_{s, M D}=\frac{E_{t o t}-2 N_{T e} E_{C d T e}-2 N_{S} E_{C d S}-2 L_{x} L_{z} \rho}{2 L_{x} L_{z}}
$$

where the factor 2 in the numerator $2 \mathrm{~L}_{x} \mathrm{~L}_{z}$ accounts for two interfaces, and the factor 2 in the denominator $2 \mathrm{~L}_{\mathrm{x}} \mathrm{L}_{\mathrm{z}}$ means that we only calculate half of the energy because the MD geometry shown in Fig. 1(a) includes both the CdTe/CdS system and its mirror image (above the dotteddashed line), whereas Eq. (2) does not include the image contribution. In Eq. (9), only the interfacial energy, $\rho$, is unknown. Hence, $\rho$ is first determined. For convenience of use with the MD data, Eq. (2) is first rewritten as

$$
E_{s}=\frac{E_{c}}{S}+\frac{E_{f} b^{2}}{8 \pi} \frac{\gamma}{S} \ln \left(\frac{h}{r_{0}}\right)+\frac{1}{2} E_{f} \frac{2 N_{T e} \Omega_{C d T e}}{2 L_{x} L_{z}}\left\{\gamma \frac{b^{2}}{S^{2}}(1+\varepsilon)^{2} \frac{f\left(\frac{1+\varepsilon}{S} h\right)}{4 \pi\left(\frac{1+\varepsilon}{S} h\right)}+\varepsilon^{2}-\frac{2 \varepsilon b(1+\varepsilon)}{S}\right\}
$$

where $\mathrm{E}_{\mathrm{f}}=\frac{2 G_{f}}{1-v_{f}}$ is Young's modulus of the film and $\Omega_{\mathrm{CdTe}}$ is the atomic volume of CdTe bulk.

Clearly, because the CdTe film is under compression in our case, the compressive Young's modulus listed in Table I should be used in Eq. (10). For dislocation-free systems, Eq. (10) becomes

$$
E_{s}=\frac{1}{2} E_{f} \varepsilon^{2} \frac{2 N_{T e} \Omega_{C d T e}}{2 L_{x} L_{z}}
$$

Equating Eqs. (9) and (11), we obtain an interfacial energy of $\rho=0.01766 \mathrm{eV} / \AA^{2}$ from the MD data for the dislocation-free systems.

When dislocations are present, Eq. (10) involves three unknown parameters $r_{0}, E_{c}, \gamma$. The dislocation core radius and core energy determined above are for homogeneous CdTe and CdS 
compounds, and it is reasonable that these parameters changed in the inhomogeneous system. As we discussed above, a large core radius always satisfies linear elastic theory, and hence we set $\mathrm{r}_{0}$ to the core radius of CdTe, $\mathrm{r}_{0}=12.4596 \AA$, which is larger than that of CdS. Under these conditions, only $\mathrm{E}_{\mathrm{c}}$ and $\gamma$ are left as unknowns. We now compare our modified continuum model, Eq. (10) or equivalently, Eq. (2), and the conventional model, Eq. (1), in terms of matching the MD data.

The results for the energy as a function of film thickness $h$ at different dislocation spacings, as obtained from the MD simulations and the conventional model, Eq. (1), are first examined in Fig. 5(a). For this case, as a clear example of the consequence for not appropriately defining the correct Burgers vector and dislocation spacing, it is assumed that the Burgers vector is defined by the film lattice and dislocation spacing defined by the substrate. Note that here the continuum model Eq. (1) is adjusted by including the dislocation core energy of CdTe. This improves the agreement of the continuum model with the MD data. It can be seen from Fig. 5(a) that for the dislocation free systems ( $\mathrm{S}$ = infinity), and the systems where misfit dislocations almost exactly cancel the mismatch strain (i.e., the $\mathrm{S}=33 \AA$ where the energy becomes almost independent of the film thickness $\mathrm{h}$ ), the agreement between the continuum and the MD models is very good. However, for films that do contain dislocations, but with the residual mismatch strain nonzero, the continuum model deviates from the MD model rather significantly.

It is not surprising that the agreement is good for dislocation free systems, since the error for the conventional models is associated with the dislocation descriptions. The incorrect definitions of Burgers vector and dislocation spacing result in a significant error of the overall strain (and hence strain energy density) of the dislocation-containing film. This accounts for the disagreement at the intermediate dislocation spacing. The conventional model also involves 
approximation $f(x) \approx 4 \pi x$. The error caused by this approximation in part cancels the error caused by the wrong strain calculation, coincidently resulting in a good agreement at the shortest dislocation spacing.

(a) Eq. (1) plus dislocation core energy

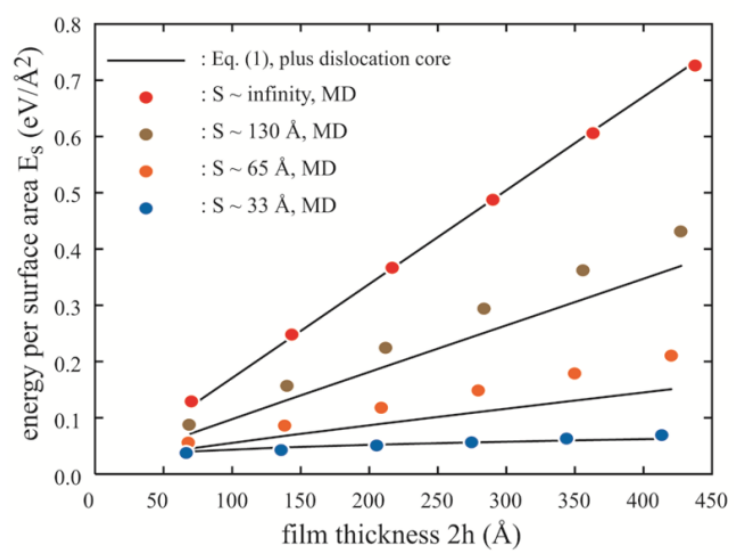

(c) Eq. (2), without optimization

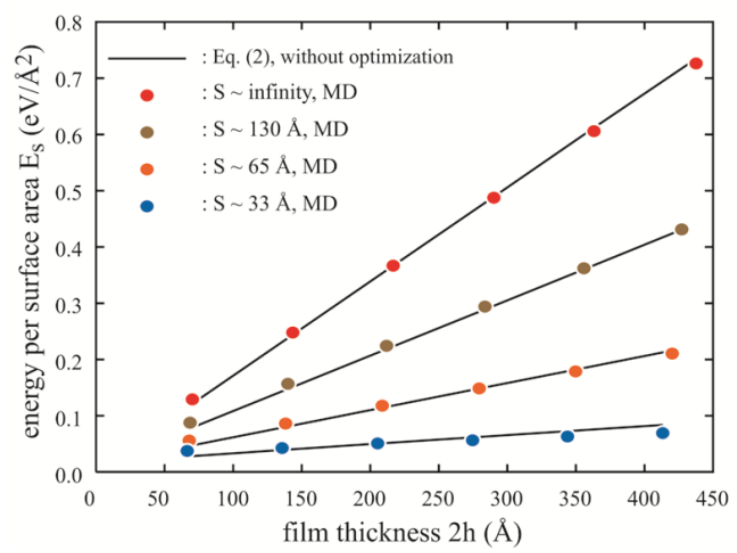

(b) Eq. (4)

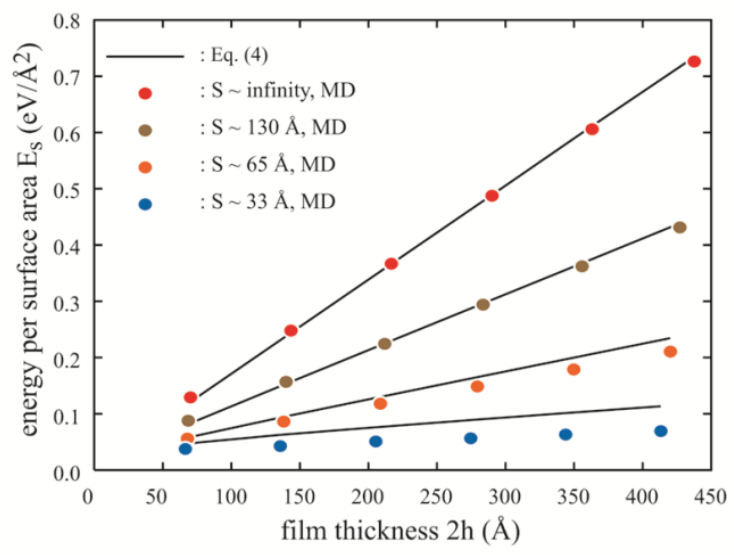

(d) Eq. (2), with optimization

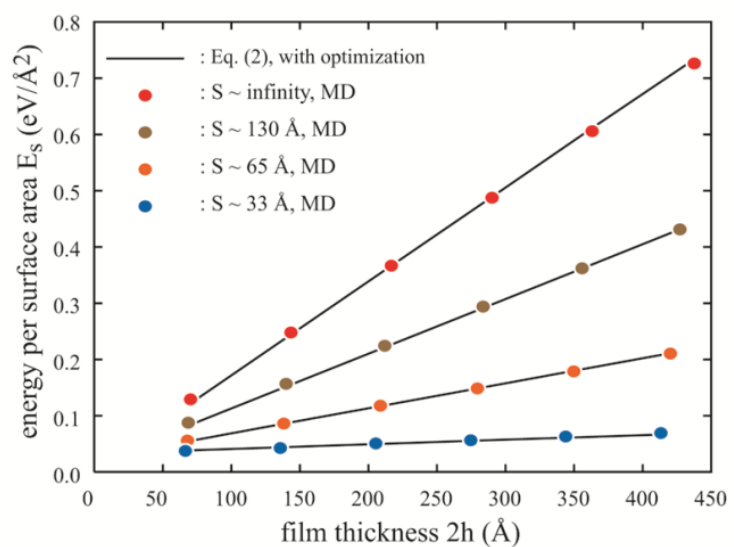

Fig. 5. Comparison between the MD and continuum models: (a) the conventional model Eq. (1) plus dislocation core energy; (b) simplified new model, Eq. (4), which use the correct values of Burgers magnitude $b$ and dislocation spacing $\mathrm{S}_{\mathrm{f}} ;(\mathrm{c})$ accurate $\mathrm{f}(\mathrm{x})$ instead of $4 \pi x$, Eq. (2), without any optimization, i.e., $\gamma=1$, and $\mathrm{E}_{\mathrm{c}}=1.2984 \mathrm{eV} / \AA \mathrm{A}$ (CdTe value); and (d) accurate $f(x)$ instead of $4 \pi x$, Eq. (2), with optimized parameters $\gamma=0.8956$ and $\mathrm{E}_{\mathrm{c}}=1.8726 \mathrm{eV} / \AA$.

Step by step, we now insert the appropriate Burgers magnitude $b_{s}$ and dislocation spacing $S_{f}$

in Eq. (1). This is essentially the simplified new model described by Eq. (4). Similar MD and continuum data are shown in Fig. 5(b). It can be seen that significant improvement is achieved as 
long as dislocations are not too close, say $\mathrm{S}>65 \AA$. The biggest disagreement occurs at the shortest dislocation spacing. Note that because the error caused by the use of the incorrect dislocation spacing and Burgers vector is removed, the error caused by the $\mathrm{f}(\mathrm{x}) \approx 4 \pi \mathrm{x}$ approximation cannot be canceled. As a result, the error at the shortest dislocation spacing seen in the improved model, Fig. 5(b), appears to be larger than that seen in the conventional model, Fig. 5(a).

Now we examine the more accurate model, Eq. (2) or equivalently Eq. (10). Eq. (2) involves two parameters $\gamma$ and $\mathrm{E}_{\mathrm{c}}$. We first test the validity of the model without fitting these two parameters, i.e., we simply set $\gamma=1$ and $\mathrm{E}_{\mathrm{c}}$ to be the CdTe value of $1.2984 \mathrm{eV} / \mathrm{A}$. These essentially mean that the overall elastic and core behavior of the film/substrate system is dominated by the film. The results obtained from the MD simulations and continuum calculations are shown in Fig. 5(c). Clearly, the new model further improves over the model shown in Fig. 5(b). The good agreement achieved at $\gamma=1$ partially supports the conventional model which only uses elastic properties of the film. From Eq. (2) or (10), the elastic inhomogeneity only affects the dislocation energy, but not the uniaxial mismatch strain energy in the film. It is therefore not surprising that the results are primarily determined by the elastic properties in the film.

Without fitting any parameters, Fig. 5(c) strongly validates our new continuum model. Now we fully optimize our model by fitting $\gamma$ and $\mathrm{E}_{\mathrm{c}}$ to the MD data. Our optimization leads to $\gamma=$ 0.8956 , and $\mathrm{E}_{\mathrm{c}}=1.8726 \mathrm{eV} / \mathrm{A}$. This fully optimized new continuum model is compared with the MD results in Fig. 5(d). Here the lines calculated from the continuum model are truly indistinguishable from the MD data, justifying making the $\gamma$ and $\mathrm{E}_{\mathrm{c}}$ parameters flexible. In particular, at very small film thickness $\sim 70 \AA$, the MD energy trends of different dislocation 
spacings are exactly reproduced by the continuum model. This is improved over Fig. 5(c). Accuracy at small film thickness is important for accurately determining the critical film thickness for dislocation formation. For example, Fig. 5 indicates that energies of the dislocationcontaining systems are lower than those of dislocation-free systems even at the smallest $2 \mathrm{~h}$ value explored ( $70 \AA)$, indicating that the critical thickness $h$ is below $35 \AA$.

It is worth noting that the condition used in MD is not exactly the same as that used in the continuum model. In particular, in order to mimic the problem of a film on a semi-infinite substrate, we do not address the interaction of dislocations in different periodic MD cells along the $y$ - direction. At the same time, we effectively mitigate the effect by fixing the MD x- and zdimensions to the CdS sizes to keep the uniaxial strain field from propagating across the $\mathrm{CdS}$ periodic boundaries. It would be interesting to release the fixed boundary condition while significantly increasing the $y$ - length of the CdS substrate (say two orders of magnitude). Unfortunately, this would require enormous computing resources. One significant impact of our work, however, is the creation of a robust MD approach that can guide the development of a continuum model for mismatched nanostructures, where the continuum theories are relatively immature, but the MD can more precisely represent the real structures. This means that the future continuum models for new misfit structures (e.g., core/shell particles) can be tuned and improved as they are being developed, because MD validation can be performed step by step with the progression of the continuum models, as opposed to much slower experimental validation.

While being more accurate, our model is just as easy to apply as the conventional model. In fact, the only complexity introduced in Eq. (2) or Eq. (10) as compared to Eq. (1) is the oneargument function $\mathrm{f}(\mathrm{x}) /(4 \pi \mathrm{x})$ where $\mathrm{x}=(1+\varepsilon) \mathrm{h} / \mathrm{S}$. As for the unknown parameter $\gamma$, the condition $\gamma=1$ is a reasonable approximation. It simply means that the strain energy is predominantly 
determined by the elastic properties of the film due to the fact that uniaxial strain energy is proportional to the square of strain and the far field strain in the substrate is assumed to be zero. As for the unknown parameter core energy $E_{c}$, since the old model neglects this term, any reasonable value is likely to improve the results. Regardless, the robust MD approach demonstrated here can always be used to determine these parameters. In that case, our new continuum model can be made indistinguishable from the MD methods. Finally, we emphasize that the studies presented in this paper are rigorous only for mirror imaged interfaces rather than true surfaces. We are currently applying the same approach as established here to examine the case of free surfaces.

\section{CONCLUSIONS}

A systematic study combining continuum calculations and MD simulations has been performed to examine the misfit dislocation formation and strain relaxation problem. The numerous insights made are useful for future research of lattice-mismatched systems where defect density and strain relaxation critically determine the performance. These are summarized below:

1. Time averaged MD simulations can produce much more accurate results (essentially zero standard deviation considering three decimal points based on an average time of $>10 \mathrm{~ns}$ ). This enables MD to be confidently used to validate continuum theories.

2. Continuum expressions of energies have been rigorously derived for both latticemismatched systems and periodic dislocation dipole arrays. The physics insight gained during the derivation of the former expression allows the continuum model of misfit dislocation formation to be improved. The latter expression allows the dislocation core radius and the core energy to be accurately calculated from MD simulations using exactly 
the same dipole array configurations without having to impose continuum boundary conditions - an approximation commonly used in the past to extend the MD length scales.

3. The dislocation spacing (S) used in the continuum theory should not be measured in the deformed system, but rather measured in the unstrained film. The magnitude of Burgers vector should not be measured in the film, but rather measured in the substrate. In a related notion, the lattice mismatch parameter should be defined as $\varepsilon=\left(a_{s}-a_{f}\right) / a_{f}$ rather than $\varepsilon=\left(a_{f^{-}}\right.$ $\left.a_{s}\right) / a_{s}$. Misuse of any of these parameters causes significant errors when the lattice mismatch of the system is large. Our new model, Eq. (2) or (10), accounts for these, as well as dislocation core energy and elastic inhomogeneity effects. When a more accurate dislocation energy term is used and the parameters optimized, the continuum calculations using Eq. (2) or (10) are essentially indistinguishable from the MD simulations.

4. Lattice-mismatched systems contain elastic constants from the two materials. Our work confirms that the conventional continuum theory, which typically uses only the film elastic constants, can remain accurate at least for the $\mathrm{CdTe} / \mathrm{CdS}$ system explored.

5. We found that the dislocation contribution to the energy of lattice-mismatched systems is $\frac{G_{f} b^{2}}{4 \pi\left(1-v_{f}\right)} \gamma \ln \left(\frac{h}{r_{0}}\right)+\frac{G_{f} b^{2}}{\left(1-v_{f}\right)} \gamma \frac{h}{S_{f}}$, rather than $\frac{G_{f} b^{2}}{4 \pi\left(1-v_{f}\right)} \gamma \ln \left(\frac{h}{r_{0}}\right)$ alone. The additional $\frac{G_{f} b^{2}}{\left(1-v_{f}\right)} \gamma \frac{h}{S_{f}}$ term accounts for dislocation interaction energy. While it does not change the overall expression of Eq. (1), our new interpretation of dislocation energy allows Eq. (1) to consistently reduce to dislocation energy when applied for homogeneous materials (i.e., the system has dislocations but vanishing mismatch strain, $\varepsilon=0$ ); 
6. The robust approach outlined here provides a theoretical means (MD) to quickly validate future continuum models for new misfit structures (e.g., core/shell particles).

\section{ACKNOWLEDGEMENTS}

Sandia National Laboratories is a multiprogram laboratory managed and operated by Sandia Corporation, a wholly owned subsidiary of Lockheed Martin Corporation, for the US

Department of Energy's National Nuclear Security Administration under contract DE-AC04-

94AL85000. This work was performed under a DOE Project No. EE0005958, and under a

Laboratory Directed Research and Development (LDRD) project 165724.

\section{References}

1 R. M. Farrell, E. C. Young, F. Wu, S. P. DenBaars, and J. S. Speck, Semicond. Sci. Technol., 27, 024001 (2012).

2 J. W. Matthews, and A. E. Blakeslee, J. Crystal Growth, 27, 118 (1974).

3 W. D. Nix, Metall. Trans. A, 20, 2217 (1989).

4 J. R. Willis, S. C. Jain, and R. Bullough, Phil. Mag. A, 62, 115 (1990).

5 X. Feng, and J. P. Hirth, J. Appl. Phys., 72, 1386 (1992).

6 J. P. Hirth, and X. Feng, J. Appl. Phys., 67, 3343 (1990).

7 S. C. Jain, T. J. Gosling, J. R. Willis, R. Bullough, and P. Balk, Solid-State Electron., 35, 1073 (1992).

8 T. J. Gosling, J. R. Willis, R. Bullough, and S. C. Jain, J. Appl. Phys., 73, 8297 (1993).

9 U. Jain, S. C. Jain, J. Nijs, J. R. Willis, R. Bullough, R. P. Mertens, and R. van Overstraeten, Solid-State Electron., 36, 331 (1993).

10 S. V. Bobylev, I. A. Ovid'ko, and A. G. Sheinerman, Phys Rev. B, 64, 224507 (2001).

11 I. A. Ovid'ko, and A. G. Sheinerman, Phys. Rev. B, 66, 245309 (2002).

12 A. J. Vattre, and M. J. Demkowicz, Comp. Mater. Sci., 88, 110 (2014).

13 A. J. Vattre, and M. J. Demkowicz, Acta Mater., 61, 5172 (2013).

14 G. Gutekunst, J. Mayer, V. Vitek, and M. Rühle, Phil. Mag. A, 75, 1357 (1997).

15 J. P. Hirth, J. Phys. Chem. Solids, 55, 985 (1994).

16 J. P. Hirth and J. Lothe, Theory of Dislocations (McGraw-Hill, New York, 1968)

17 D. Maroudas, L. A. Zepeda-Ruiz, and W. H. Weinberg, Surf. Sci., 411, L865 (1998).

18 A. P. Payne, W. D. Nix, B. M. Lairson, and B. M. Clemens, Phys. Rev. B, 47, 13730 (1993).

19 L. Pizzagalli, G. Cicero, and A. Catellani, Phys. Rev. B, 68, 195302 (2003).

20 L. Dong, J. Schnitker, R. W. Smith, and D. J. Srolovits, J. App. Phys. 83, 217 (1993).

21 J. Godet, L. Pizzagalli, S. Brochard, and P. Beauchamp, Phys. Rev. B, 70, 054109 (2004).

22 A. Ouahab, C. Mottet, and J. Goniakowski, Phys. Rev. B, 72, 035421 (2005).

23 K. Wiesauer, and G. Springholz, Phys. Rev. B, 69, 245313 (2004). 
24 A. J. Vattre, N. Abdolrahim, K. Kolluri, and M. J. Demkowicz, Sci. Rep., 4, 6231 (2014).

25 X. Zhou, D. K. Ward, B. M. Wong, F. P. Doty, and J. A. Zimmerman, J. Phys. Chem. C, 116,17563 (2012).

26 S. Pattamatta, R. S. Elliott, and E. B. Tadmor, Proc. Nat. Acad. Sci., 111, E1678 (2014).

27 X. W. Zhou, S. Aubry, R. E. Jones, A. Greenstein, and P. K. Schelling, Phys. Rev. B, 79, 115201 (2009).

28 Publisher to provide a link.

29 J. H. van der Merwe, J. Appl. Phys., 34, 123 (1963).

30 J. W. Matthews, and A. E. Blakeslee, J. Crystal Growth, 29, 273 (1975).

31 J. W. Matthews, J. Vac. Sci. Technol., 12, 126 (1975).

32 U. Jain, S. C. Jain, J. Nijs, J. R. Willis, R. Bullough, R. P. Mertens, and R. van Overstraeten, Solid-State Electron., 36, 331 (1993).

33 U. Jain, S. C. Jain, J. Nijs, J. R. Willis, R. Bullough, R. P. Mertens, and R. van Overstraeten, Solid-State Electron., 36, 331 (1993).

34 J. W. Matthews, and A. E. Blakeslee, J. Crystal Growth, 27, 118 (1974).

35 U. Jain, S. C. Jain, J. Nijs, J. R. Willis, R. Bullough, R. P. Mertens, and R. van Overstraeten, Solid-State Electron., 36, 331 (1993).

36 S. C. Jain, T. J. Gosling, J. R. Willis, D. H. J. Totterdell, and R. Bullough, Phil. Mag. A, 65, 1151 (1992).

37 S. C. Jain, A. H. Harker, and R. A. Cowley, Phil. Mag. A, 75, 1461 (1997).

38 W. Cai, V. V. Bulatov, J. Chang, J. Li, and S. Yip, Phys. Rev. Lett., 86, 5727 (2001).

39 X. W. Zhou, D. K. Ward, J. E. Martin, F. B. van Swol, J. L. Cruz-Campa, and D. Zubia, Phys. Rev. B, 88, 085309 (2013).

40 E. B. Webb III, J. A. Zimmerman, and S. C. Seel, Math. Mech. Solids, 13, 221 (2008). 